\title{
Probabilistic Fault Diagnosis in the MAGNETO Autonomic Control Loop
}

\author{
Pablo Arozarena ${ }^{1}$, Raquel Toribio ${ }^{1}$, Jesse Kielthy ${ }^{2}$, Kevin Quinn $^{2}$, and Martin Zach $^{3}$ \\ ${ }^{1}$ Telefónica Investigación y Desarrollo, Madrid, Spain \\ \{pabloa, raquelt\} atid.es \\ ${ }^{2}$ Telecommunications Software and Systems Group (TSSG), Waterford, Ireland \\ \{jkielthy, kquinn\} atssg.org \\ ${ }^{3}$ Siemens AG Austria, Viena, Austria \\ \{martin.zach\} asiemens.com
}

\begin{abstract}
Management of outer edge domains is a big challenge for service providers due to the diversity, heterogeneity and large amount of such networks, together with limited visibility on their status. This paper focuses on the probabilistic fault diagnosis functionality developed in the MAGNETO project, which enables finding the most probable cause of service problems and thus triggering appropriate repair actions. Moreover, its self-learning capabilities allow continuously enhancing the accuracy of the diagnostic process.
\end{abstract}

Keywords: Autonomic, Home Area Networks (HAN), Probabilistic Management, Bayesian Network, Self-learning.

\section{Introduction}

As networks grow in size, heterogeneity, and complexity, network management needs to deal with incomplete management information, uncertain situations, lack of full visibility on network status and dynamic changes. All this is particularly true for outer edge domains, i.e. the point of attachment of home area networks (HANs).

To address these problems, several new management approaches have been developed. Current autonomic management architectures have adapted the initial IBM concept [1] to enable self management of networks. For example, FOCALE [2] was designed with the concept of autonomic network management in mind and considers the additional constraint of heterogeneity of devices. Moreover, the Generic Autonomic Networking Architecture (GANA) [3] provides a framework to model how protocols, functions, nodes and networks can provide autonomic network management. In order to deal with uncertainty, other approaches have explored the application of probabilistic techniques to network management. CAPRI [4] uses Bayesian Network (BN) inference [5], combined with a highly distributed architecture, to conclude the cause of the observed network problems. The 4WARD project [6] has proved the feasibility of probabilistic techniques to address the composition of management functions in distributed architectures.

MAGNETO [7], a project funded under the Eureka Celtic Initiative, is addressing the challenges described above in the particular case of Home Area Networks (HANs). The approach in MAGNETO is to deploy management functionality both 
inside HANs and in the ISP domain to enable distributed autonomic behavior, thus allowing management tasks to span different domains. The combination of shared autonomic management produces additional benefits - for example, if a problem in the access network is affecting several HANs, MAGNETO agents at the ISP are in a better position to properly diagnose the fault. MAGNETO addresses two main use cases: Service Degradation - which focuses on how a network can react to degradation in the quality of a service, and Service Breakdown - which focuses on how a network can react to problems disrupting the delivery of a given service.

\section{Fault Diagnosis in MAGNETO}

Fault diagnosis in MAGNETO follows a distributed approach that, based on probabilistic techniques, allows the system to face uncertainty. In MAGNETO, the possible causes of service failures (hypothesis) detected in the network and the set of observable network variables (symptoms) are modeled as a BN. In this BN possible causes are defined as hypothesis nodes while symptoms as evidence nodes. Thanks to bayesian inference, the probability of each of the possible causes of a service failure can be estimated. Thus, the result of the diagnosis procedure is a set of the most probable causes of the failure together with their probability values. In MAGNETO, the initial structure and Conditional Probability Tables (CPTs) of the BN are modeled with the help of the network/service experts.

Since MAGNETO deals with the management of services in the outer edge, the causes of problems may lie in different domains (such as the HAN, the ISP, etc.). For this reason, MAGNETO relies on a Multi-Agent System (MAS) [8] platform that facilitates distributing fault diagnosis functionality across domains. Besides, MAGNETO allows breaking down a BN in a set of smaller BNs that can be used by agents spread across the different network domains. For instance, some agents may be specialized in diagnosing HAN errors while others in diagnosing access network errors, and they may exchange their results to cooperatively reach a global diagnosis. The following types of agents have been defined:

- Diagnosis Agents orchestrate the diagnostic process among a set of cooperating agents. This algorithm is an iterative process where new evidences are added to the $\mathrm{BN}$ as they become available and bayesian inference is repeatedly performed until enough confidence for a given hypothesis is reached or no extra information can be obtained. In the latter case, the diagnosis can be delegated to a different diagnosis agent for further inference.

- Observation agents provide evidences which are obtained on request by performing specific tests, such as alarm queries. Each observation agent is specialised in a certain type of tests and publishes its capabilities in a service directory.

- Belief Agents provide the set of probability values of a given node being in each of its possible states. These agents have bayesian knowledge embedded and perform bayesian inference to obtain the beliefs of a certain node based on the evidence they may get from observation agents. By exchanging beliefs, these agents enable partitioning a $\mathrm{BN}$ to perform distributed diagnosis.

- A Knowledge Agent is in charge of conducting a self-learning process and distributing diagnosis knowledge to other agents. 
An important MAGNETO goal is to be able to automatically improve the quality of its diagnosis results. In order to do so, past diagnosis reports should be validated either automatically or manually. At the time of writing, only manual validation is supported since automatic validation requires feeding back to the fault diagnosis functionality the results of autonomic self healing actions performed as a consequence of a given diagnosis. Once MAGNETO has enough new validated reports, it triggers a parametric bayesian learning algorithm, called Expectation Maximization (EM) [9], to obtain more accurate CPTs. In order to perform self-learning, MAGNETO periodically executes the following steps:

1. Diagnosis agents send diagnosis operation reports to the knowledge agent. These reports contain the results of diagnostic operations and are stored in a knowledge repository.

2. Diagnosis reports are validated to indicate whether the result was right or wrong. This validation can be either manual or automatic.

3. A self-learning process is executed by the knowledge agent. This process accesses the knowledge repository and updates CPT values using the validated diagnosis results and the $\mathrm{BN}$ knowledge available at that point in time. It is important to note that a confidence value is used by the self-learning algorithm to determine the weight of the existing $\mathrm{BN}$ parameters over new incoming data.

4. Once new CPT values are learnt, they are propagated to all diagnosis agents so they can update their inference knowledge accordingly.

\section{Conclusions and Future Work}

The MAGNETO prototype is being validated in a real testbed representing two interconnected HANs and an ISP. Feedback from this validation, together with further research outputs, will be used to define the evolution of the prototype. Future work related to fault diagnosis will mainly focus on the following research topics:

- Studying ways to automatically validate diagnosis results so human intervention can be avoided, thus making the self-learning loop more efficient.

- Using a different confidence value per each parameter in a BN to make the selflearning process more accurate.

- Comparing different ways to combine self-learning with BN partitioning in terms of facility to cope with different domains, accuracy of results, simplicity of the validation process, etc.

\section{Acknowledgments}

This paper describes work undertaken in the context of the CELTIC MAGNETO project, which is partially funded by the Spanish "Ministerio de Industria, Turismo y Comercio" under the Avanza program, by "Enterprise Ireland" as part of the International Collaboration Support Programme and by the Austrian FFG (Basisprogramme, Projekt Nr. 820360). 


\section{References}

1. Kephart, J.O., Chess, D.M.: The Vision of Autonomic Computing. Computer 36(1), 41-50 (2003)

2. Strassner, J., Agoulmine, N., Lehtihet, E.: Focale: A novel autonomic networking architecture. In: Latin American Autonomic Computing Symposium (LAACS), Campo Grande, MS, Brazil (2006)

3. Chaparadza, R., et al.: Creating a viable Evolution Path towards Self-Managing Future Internet via a Standardizable Reference Model for Autonomic Network Engineering. In: Towards the Future Internet - A European Research Perspective, pp. 136-147. IOS Press, Amsterdam (2009)

4. Lee, G.: Capri: A common architecture for distributed probabilistic internet fault diagnosis. Massachusetts Institute of Technology. Dept. of Electrical Engineering and Computer Science. Thesis, Ph. D. (2007)

5. Barco Moreno, R.: Bayesian modelling of fault diagnosis in mobile communication networks. Universidad de Málaga, Tech. Rep. (2007)

6. Hasslinger, G., et al.: 4WARD Deliverable D-4.2: In-Network Management Concept (2009), http: / /www . 4ward-project. eu

7. MAGNETO: Management of the outer edge, http: / /projects.celtic-initiative.org/MAGNETO

8. Wooldridge, M.: An Introduction to Multi Agent Systems, 2nd edn. John Wiley \& Sons, Chichester (2009)

9. Hastie, T., Tibshirani, R., Friedman, J.: The Elements of Statistical Learning, pp. 236-243. Springer, Heidelberg (2001) 